\title{
WEAK UCP AND PERTURBED MONOPOLE EQUATIONS
}

\author{
B. BOOSS-BAVNBEK, M. MARCOLLI ${ }^{\dagger}$, AND B.L. WANG ${ }^{\ddagger}$
}

\begin{abstract}
We give a simple proof of weak Unique Continuation Property for perturbed Dirac operators, using the Carleman inequality. We apply the result to a class of perturbations of the Seiberg-Witten monopole equations that arise in Floer theory.
\end{abstract}

\section{INTRODUCTION}

We outline the content and motivation of the paper. The first part of the paper presents a proof of weak Unique Continuation Property for perturbed Dirac operators, based on classical methods revolving around the Carleman inequality. In the second part we give an application to the Morse-Smale-Witten complex for the Chern-Simons-Dirac functional, whose homology defines a gauge-theoretic invariant of 3-manifolds.

1.1. UCP and Dirac operators. A linear or non-linear operator $\mathfrak{D}$, acting on functions or sections of a bundle over a compact or non-compact manifold $M$ has the weak Unique Continuation Property (UCP) if any solution $u$ of the equation $\mathfrak{D} u=0$ has the following property: if $u$ vanishes on a non-empty open subset $\Omega$ of $M$, it vanishes on the whole connected component of $M$ containing $\Omega$.

There is also a notion of strong UCP, where, instead of assuming that a solution $u$ vanishes on an open subset, one assumes only that $u$ vanishes 'of high order' at a point. The concepts of weak and strong UCP extend a fundamental property of analytic functions to some elliptic equations other than the Cauchy-Riemann equation, see also $\$ 2.2$ below.

Up to now, (almost) all work on UCP goes back to two seminal papers [6], [7] by Torsten Carleman, establishing a Carleman-type inequality (cf. our inequalities (2.3) and (4.1) below). In this approach, the difference between weak and strong UCP and the possible presence of more delicate non-linear perturbations are related to different choices of the weight function in the inequality, and to whether $L^{2}$-estimates suffice or $L^{p}-$ and $L^{q}-$ estimates are required.

\footnotetext{
${ }^{\dagger}$ Partially supported by Humboldt Foundation Sofja Kovalevskaja Award.

${ }^{\ddagger}$ Supported by Australian Research Council.
} 
There are different notions of operators of Dirac type. We shall not discuss the original hyperbolic Dirac operator (in the Minkowski metric), but restrict ourselves to the elliptic case related to Riemannian metrics.

Recall that, if $(M, g)$ is a compact smooth Riemannian manifold (with or without boundary) with $\operatorname{dim} M=m$, we denote by $\mathfrak{C l}(M)=\left\{\mathfrak{C} l\left(T M_{x}, g_{x}\right)\right\}_{x \in M}$ the bundle of Clifford algebras of the tangent spaces. For $E \rightarrow M$ a smooth complex vector bundle of Clifford modules, the Clifford multiplication is a bundle map c : $\mathfrak{C} l(M) \rightarrow \operatorname{Hom}(E, E)$ which yields a representation $\mathbf{c}: \mathfrak{C} l\left(T M_{x}, g_{x}\right) \rightarrow \operatorname{Hom}_{\mathbb{C}}\left(E_{x}, E_{x}\right)$ in each fiber. We may assume that the bundle $E$ is equipped with a Hermitian metric which makes the Clifford multiplication skew-symmetric

$$
\left\langle\mathbf{c}(v) s, s^{\prime}\right\rangle=-\left\langle s, \mathbf{c}(v) s^{\prime}\right\rangle \quad \text { for } v \in T M_{x} \text { and } s \in E_{x} .
$$

Any choice of a smooth connection

$$
\nabla: \mathrm{C}^{\infty}(M ; E) \rightarrow \mathrm{C}^{\infty}\left(M ; T^{*} M \otimes E\right)
$$

defines an operator of Dirac type $\mathfrak{D}:=\mathbf{c} \circ \nabla$ under the Riemannian identification of the bundles $T M$ and $T^{*} M$. In local coordinates we have $\mathfrak{D}:=\sum_{j=1}^{m} \mathbf{c}\left(e_{j}\right) \nabla_{e_{j}}$ for any orthonormal base $\left\{e_{1}, \ldots, e_{m}\right\}$ of $T M_{x}$. Actually, we may choose a local frame in such a way that

$$
\nabla_{e_{j}}=\frac{\partial}{\partial x_{j}}+\text { zero order terms }
$$

for all $1 \leq j \leq m$. So, locally, we have

$$
\mathfrak{D}:=\sum_{j=1}^{m} \mathbf{c}\left(e_{j}\right) \frac{\partial}{\partial x_{j}}+\text { zero order terms . }
$$

It follows at once that the principal symbol $\sigma_{1}(\mathfrak{D})(x, \xi)$ is given by Clifford multiplication with $i \xi$, so that any operator $\mathfrak{D}$ of Dirac type is elliptic with symmetric principal symbol. Actually, if the connection $\nabla$ is compatible with Clifford multiplication (i.e. $\nabla \mathbf{c}=0$ ), then the operator $\mathfrak{D}$ itself becomes symmetric. We shall, however, admit non-compatible metrics. Moreover, the Dirac Laplacian $\mathfrak{D}^{2}$ has principal symbol $\sigma_{2}\left(\mathfrak{D}^{2}\right)(x, \xi)$ given by the Riemannian metric $\|\xi\|^{2}$. So, it is scalar real (i.e. a real multiple of the identity) and elliptic.

1.2. Motivation. Our main motivation for investigating weak UCP for perturbed Dirac equations is an application to gauge theory of 3-manifolds. We outline briefly the context in which the question arises.

Seiberg-Witten Floer homology is an invariant of 3-manifolds defined as the homology of a Morse-Smale-Witten complex for the Chern-Simons-Dirac functional, defined on an infinite dimensional space of $U(1)$-connections and spinor sections. A detailed construction of Seiberg-Witten Floer homology along with an analysis of its main properties is given in [14]. 
Several interesting analytical problem are connected to the construction of this invariant. One source of technical difficulties is finding a suitable perturbation theory for the functional, in order to have the Morse (or Morse-Bott) condition for the critical points, and transversality of the spaces of flow lines. There are different ways of treating this problem.

One possible approach is the one followed in [14]. First one realizes that it is fairly easy to achieve transversality for the set of critical points of the Chern-Simons-Dirac functional (moduli space of gauge classes of 3-dimensional Seiberg-Witten monopoles), while it is more difficult to achieve transversality for moduli spaces of flow lines. This observation leads to the idea of perturbing the functional just enough to achieve transversality at critical points, and then introducing a class of perturbation of the $4-$ dimensional Seiberg-Witten monopole equations, which does not come from a perturbation of the functional. The perturbations constructed this way have to satisfy a certain list of properties [14, §2.3], ensuring that the resulting moduli spaces of flow lines have the desired transversality properties, so that the boundary operator in the Morse-Smale-Witten complex can be defined by a counting of flow lines.

In this approach, all the perturbations can be chosen so that, in the Seiberg-Witten monopole equations, only the curvature equation is perturbed, while leaving the Dirac equation unchanged. A class of perturbations with these properties was introduced by Frøyshov in [10].

Though this approach is effective in providing a working definition of Floer homology and in the proof of topological invariance (in the equivariant case) [14], it is very unnatural to use different perturbations for critical points and flow lines which do not come from a perturbation of the functional. Moreover, with the perturbation defined by Frøyshov, it is hard to have good control of the effect on the equations when the underlying 3-manifold is modified, for instance by stretching a long cylinder, as in problems related to cutting and pasting (surgery formulae). In the interest of deriving formulae of this sort, it is better to investigate other possible perturbation theories, which arise directly as perturbations of the Chern-Simons-Dirac functional. A class of such perturbation was proposed by Kronheimer in [11. Since these affect both the curvature and the Dirac equation, and are both non-local and non-linear, the question arises of how much delicate properties of the Dirac operator, such as the Unique Continuation Property, may be affected by the presence of perturbation. In particular, since the weak UCP plays a role in all the transversality arguments, this seems an important technical point that needs to be understood.

This is the main point in our paper. We apply our elementary proof of weak UCP for perturbed Dirac operators to a class of perturbations for Seiberg-Witten Floer theory that combines the perturbations introduced in [5] and [11]. 
Finally, it should be mentioned that a version of Seiberg-Witten Floer theory that avoids the use of perturbations and deals directly with the resulting excess intersection was developed recently by Manolescu [13].

Acknowledgment. We thank Hubert Kalf for his generous help and many extremely useful comments and suggestions.

\section{WEAK UCP FOR PERTURBED DiRAC OPERATORS}

Clearly, not any arbitrary perturbation by a 0th order term preserves the Unique Continuation Property (UCP) as one can see by standard cases from first order ordinary differential equations, such as $\dot{u}-2 \sqrt{|u|}=0$, or $\dot{u}-3 u^{2 / 3}=0$. With a little more work, it is possible to produce examples with non-uniqueness of smooth solutions.

In the affirmative, weak UCP can be proved in the following case.

Theorem 2.1. The weak UCP (i.e. UCP from open subsets) for solutions of a possibly tensored Dirac equation $\emptyset_{\mathrm{A}} u=0$ on a smooth (not necessarily compact) manifold $M$ is preserved under perturbation with a non-linear and/or global term of 0th order of the form $\mathfrak{P}(u)$ which can be estimated in the following way:

- $|\mathfrak{P}(u)(x)| \leq P(u, x)|u(x)|$ with a real valued non-negative locally bounded function $P(u, \cdot)$ on $M$.

2.1. Guide to the Literature. We review briefly three ways to prove Theorem 2.1.

2.1.1. We can replace the Dirac operator by its square, the Dirac Laplacian. It has diagonal and real principal symbol. So we can apply the legendary parallel papers [1] and [8] by Aronszajn and Cordes. Of course, in principle, a perturbation of a differential operator of first order by a 0th order term is essentially more delicate than a perturbation of a second order operator by 0th order. Fortunately, the two mentioned papers admit non-linear perturbations of first order of precisely that type which our 0th order perturbation of the Dirac operator will yield by squaring the perturbed operator.

Strictly speaking, Aronszajn and Cordes derived their results only for scalar equations and not for systems, but they mentioned that the proofs are similar for systems. Moreover, it should be mentioned that these methods are extremely hard because the goal of the two famous papers was the strong UCP (from a point). However, for the applications in Seiberg-Witten-Floer theory, weak UCP suffices. Finally, from a geometric point of view it seems inappropriate to destroy the beautiful geometric first-order structure of the Dirac operator by squaring.

2.1.2. For weak UCP, an alternative and much simpler proof was given by Weck, 18] for any first order system of generalized Dirac type and a non-linear smooth perturbation satisfying the condition of Theorem 2.1. He transforms to a particular second order system and then establishes weak UCP for that system relatively easily. 
2.1.3. In the following we shall explain a third and completely direct proof method, where all arguments are carried out on the level of the perturbed Dirac operator, without resorting to second order operators.

First we recall some features of the direct proof of weak UCP for solutions of the Dirac equation, given in [2] and [3]. Later on we explain the modifications necessary to cover also the non-linear case.

2.2. The Unique Continuation Property - The Unperturbed Case. The weak $\mathrm{UCP}$ is one of the basic properties of an operator of Dirac type $\not_{\mathrm{A}}$. For $M=M_{0} \cup_{\Sigma} M_{1}$, it guarantees that there are no ghost solutions of $\emptyset_{\mathrm{A}} u=0$, that is, there are no solutions which vanish on $M_{0}$ and have non-trivial support in the interior of $M_{1}$. This property is also called UCP from open subsets or across any hypersurface. For Euclidean (classical) Dirac operators (i.e., Dirac operators on $\mathbb{R}^{m}$ with constant coefficients and without perturbation) the property follows by squaring directly from the well-established UCP for the classical (constant coefficients and no potential) Laplacian.

In [2, Chapter 8] a very simple proof of the weak UCP for operators of Dirac type is given, inspired by [15, Sections 6-7, in particular the proof of inequality (7.11)] and [17, Section II.3]. We refer to [3] for a further slight simplification and a broader perspective. The proof does not use advanced arguments of the Aronszajn/Cordes type regarding the diagonal and real form of the principal symbol of the Dirac Laplacian, but only the following product property of Dirac type operators (besides Gårding's inequality, see Remark 4.1).

Lemma 2.2. Let $\Sigma$ be a closed hypersurface of $M$ with orientable normal bundle. Let $t$ denote a normal variable with fixed orientation such that a bicollar neighborhood $N$ of $\Sigma$ is parameterized by $[-\epsilon,+\epsilon] \times \Sigma$. Then any operator of Dirac type can be rewritten in the form

$$
\left.\not D_{\mathrm{A}}\right|_{N}=\mathbf{c}(d t)\left(\frac{\partial}{\partial t}+B_{t}+C_{t}\right),
$$

where $B_{t}$ is a self-adjoint elliptic operator on the parallel hypersurface $\Sigma_{t}$, and $C_{t}$ : $\left.\left.S\right|_{\Sigma_{t}} \rightarrow S\right|_{\Sigma_{t}}$ a skew-symmetric operator of 0th order, actually a skew-symmetric bundle homomorphism.

Remark 2.3. (a) It is worth mentioning that the product form (2.1) is invariant under perturbation by a bundle homomorphism. More precisely: Let $\mathfrak{D}$ be an operator on $M$ which can be written in the form (2.1) close to any closed hypersurface $\Sigma$, with $B_{t}$ and $C_{t}$ as explained in the preceding Lemma. Let $R$ be a bundle homomorphism. Then

$$
\left.(\mathfrak{D}+R)\right|_{N}=\mathbf{c}(d t)\left(\frac{\partial}{\partial t}+B_{t}+C_{t}\right)+\left.\mathbf{c}(d t) S\right|_{N}
$$


with $\left.S\right|_{N}:=\left.\mathbf{c}(d t)^{*} R\right|_{N}$. Splitting $S=\frac{1}{2}\left(S+S^{*}\right)+\frac{1}{2}\left(S-S^{*}\right)$ into a symmetric and a skew-symmetric part and adding these parts to $B_{t}$ and $C_{t}$, respectively, yields the wanted form of $\left.(\mathfrak{D}+R)\right|_{N}$.

(b) For operators of Dirac type, it is well known that a perturbation by a bundle homomorphism is equivalent to modifying the underlying connection of the operator. This gives an alternative argument for the invariance of the form (2.1) for operators of Dirac type under perturbation by a bundle homomorphism.

(c) By the preceding arguments (a), respectively (b), establishing weak UCP for sections belonging to the kernel of a Dirac type operator respectively an operator which can be written in the form (2.1) implies weak UCP for all eigensections. Warning: for general linear elliptic differential operators, weak UCP for "zero-modes" does not imply weak UCP for all eigensections.

To prove the weak UCP, in combination with the preceding lemma, the standard lines of the UCP literature can be radically simplified, namely with regard to the weight functions and the integration order of estimates. In Section 2.2 we shall see that exactly these simplifications make it very easy to generalize the weak UCP to the perturbed case. We recall some decisive steps of the simplified proof in the unperturbed case.

We want to show that, if $u \in \mathrm{C}^{\infty}(M, S)$ is a solution of $\not_{\mathrm{A}} u=0$, which vanishes on a non-empty open subset $\Omega$ of $M$, then it vanishes on the whole connected component of the manifold.

2.2.1. First we localize and convexify the situation and we introduce spherical coordinates (see Figure 1 ). Without loss of generality we may assume that $\Omega$ is maximal, namely the union of all open subsets where $u$ vanishes. If the solution $u$ does not vanish on the whole connected component containing $\Omega$, we consider a point $x_{0} \in \operatorname{supp} u \cap \partial \Omega$. We choose a point $p$ inside of $\Omega$ such that the ball around $p$ with radius $r:=\operatorname{dist}\left(x_{0}, p\right)$ is contained in $\bar{\Omega}$. We call the coordinate, running from $p$ to $x_{0}$, the normal coordinate and denote it by $t$. The boundary of the ball around $p$ of radius $r$ is a hypersphere and will be denoted by $\mathcal{S}_{p, 0}$. It goes through $x_{0}$ which has a normal coordinate $t=0$.

Correspondingly, we have larger hyperspheres $\mathcal{S}_{p, t} \subset M$ for $0 \leq t \leq T$ with $T>0$ sufficiently small. In such a way we have parameterized an annular region $N_{T}:=$ $\left\{\mathcal{S}_{p, t}\right\}_{t \in[0, T]}$ around $p$ of width $T$ and inner radius $r$, ranging from the hypersphere $\mathcal{S}_{p, 0}$ which is contained in $\bar{\Omega}$, to the hypersphere $\mathcal{S}_{p, T}$ which cuts deeply into supp $u$, if $\operatorname{supp} u$ is not empty.

2.2.2. Next, we replace the solution $u$ by a cutoff

$$
v(t, y):=\varphi(t) u(t, y)
$$

with a smooth bump function $\varphi$ with $\varphi(t)=1$ for $t \leq 0.8 T$ and $\varphi(t)=0$ for $t \geq 0.9 T$. Then $\operatorname{supp} v$ is contained in $N_{T}$. More precisely, it is contained in the annular region $N_{0.9 T}$. Moreover, $\operatorname{supp}\left(\not_{\mathrm{A}} v\right)$ is contained in the annular region $0.8 T \leq t \leq 0.9 T$. 


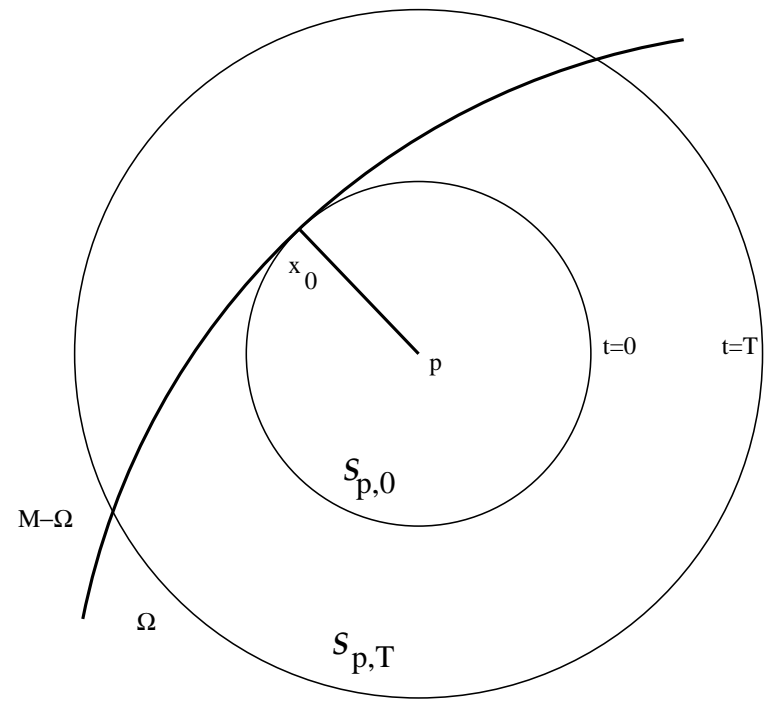

Figure 1. Local specification for the Carleman estimate

We recall the following Lemma (cf. Lemma 5 and Lemma 6 in [3]). The weak UCP for the unperturbed Dirac operator $D_{\mathrm{A}}$ will then follow immediately from this result.

Lemma 2.4. Let $\nsupseteq_{\mathrm{A}}: \mathrm{C}^{\infty}(M, E) \rightarrow \mathrm{C}^{\infty}(M, E)$ be a linear elliptic differential operator of order 1 which can be written on $N_{T}$ in the product form (2.1). Let $v$ be a spinor section in the domain of $\not_{\mathrm{A}}$, such that $\operatorname{supp}(v) \subset N_{T}$.

(a) Then for $T$ sufficiently small there exists a constant $C$ such that the Carleman inequality (see [6], [7] for the original form)

$$
R \int_{t=0}^{T} \int_{\mathcal{S}_{p, t}} e^{R(T-t)^{2}}|v(t, y)|^{2} d y d t \leq C \int_{t=0}^{T} \int_{\mathcal{S}_{p, t}} e^{R(T-t)^{2}}\left|\not_{\mathrm{A}} v(t, y)\right|^{2} d y d t
$$

holds for any real $R$ sufficiently large.

(b) Let $u$ be a solution of $\not_{\mathrm{A}} u=0$, with $\operatorname{supp}(\varphi u) \subset N_{0.9 T}$, for $\varphi$ the cutoff function as in (2.2). If (2.3) holds for any sufficiently large $R>0$, then $u$ is equal 0 on $N_{T / 2}$.

Notice that, in the proof of (2.3) we do not assume $v$ is a solution, and we do not even assume it is smooth: the Carleman inequality is valid for any section with sufficiently small support, whether it is the cutoff of a solution of the homogeneous equation or not.

In the following section, we will return to some special features of the proof of the preceding lemma and show that the lemma remains true when an admissible (i.e. suitably bounded) perturbation is introduced. 
2.3. The Perturbed Case. We replace the equation $\not_{\mathrm{A}} u=0$ by

$$
\widetilde{D_{\mathrm{A}}} u:=\not_{\mathrm{A}} u+\mathfrak{P}_{A}(u)=0 \text {, }
$$

where $\mathfrak{P}_{A}$ is an admissible perturbation, in the following sense.

Definition 2.5. A perturbation is admissible if it satisfies the following estimate:

$$
\left|\mathfrak{P}_{A}(u)\right|_{x}|\leq P(u, x)| u(x) \mid \quad \text { for } x \in M
$$

with a real-valued function $P(u, \cdot)$ which is locally bounded on $M$ for each fixed $u$.

Example 2.6. Some typical examples of perturbations satisfying the admissibility condition of Definition 2.5 are:

- Consider a non-linear perturbation

$$
\left.\mathfrak{P}_{A}(u)\right|_{x}:=\omega(u(x)) \cdot u(x),
$$

where $\left.\omega(u(x))\right|_{x \in M}$ is a (bounded) function which depends continuously on $u(x)$, for instance, for a fixed (bounded) spinor section $a(x)$ we can take

$$
\omega(u(x)):=\langle u(x), a(x)\rangle
$$

with $\langle\cdot, \cdot\rangle$ denoting the Hermitian product in the fiber of the spinor bundle over the base point $x \in M$. This satisfies (2.5).

- Another interesting example is provided by (linear) non-local perturbations with

$$
\omega(u, x)=\left|\int k(x, z) u(z) d z\right|
$$

with suitable integration domain and integrability of the kernel $k$. These also satisfy (2.5).

- Clearly, an unbounded perturbation may be both non-linear and global at the same time. This will, in fact, be the case in our main application. In all these cases the only requirement is the estimate (2.5) with bounded $\omega(u(\cdot))$.

We now show that (admissible) perturbed Dirac operators always satisfy the weak Unique Continuation Property.

Theorem 2.7. Let $\not_{\mathrm{A}}$ be an operator of Dirac type and $\mathfrak{P}_{A}$ an admissible perturbation. Then any solution $u$ of the perturbed equation (2.4) vanishes identically on any connected component of the underlying manifold if it vanishes on a non-empty open subset of the connected component.

Proof. Let $u$ be a solution of the perturbed equation which vanishes on an open nonempty set $\Omega$. We make the same construction as in the unperturbed case with a point $x_{0} \in \Omega \cap \operatorname{supp}(u)$, a point $p$ nearby in the interior of $\Omega$, a normal coordinate $t$, hyperspheres $\mathcal{S}_{p, t}$, a small positive number $T$, a cut-off function $\varphi$ with $\varphi(t)=1$ for 
$t \leq 0.8 T$ and $\varphi(t)=0$ for $t \geq 0.9 T$. Then we consider the cut-off solution $v=\varphi \cdot u$. Note that the support of $v$ is in the interior of the hypersphere $\mathcal{S}_{p, T}$ but $v$ is not a true solution.

We argue very much like in [3, Proof of Lemma 6]. To begin with, we have

$$
\begin{aligned}
e^{R T^{2} / 4} \int_{0}^{\frac{T}{2}} \int_{\mathcal{S}_{p, t}}\|u(t, y)\|^{2} d y d t=\int_{0}^{\frac{T}{2}} & \int_{\mathcal{S}_{p, t}} e^{R T^{2} / 4}|u(t, y)|^{2} d y d t \\
& \leq \int_{0}^{T} \int_{S_{p, t}} e^{R(T-t)^{2}}|\varphi u(t, y)|^{2} d y d t=: I
\end{aligned}
$$

We apply the Carleman type inequality of Lemma 2.4

$$
I=\int_{t=0}^{T} \int_{\mathcal{S}_{p, t}} e^{R(T-t)^{2}}|\varphi u(t, y)|^{2} d y d t \leq \frac{C}{R} \int_{t=0}^{T} \int_{\mathcal{S}_{p, t}} e^{R(T-t)^{2}}\left|\not \not_{\mathrm{A}}(\varphi u)(t, y)\right|^{2} d y d t .
$$

We assume that $u$ is a solution of the perturbed equation $\not_{\mathrm{A}} u+\mathfrak{P}_{A}(u)=0$, hence

$$
\not D_{\mathrm{A}}(\varphi u)=\varphi \not_{\mathrm{A}} u+\mathbf{c}(d t) \varphi^{\prime} u=-\varphi \mathfrak{P}_{A}(u)+\mathbf{c}(d t) \varphi^{\prime} u \text {. }
$$

Inserting in (2.7) yields

$$
I \leq \frac{2 C}{R} \int_{t=0}^{T} \int_{\mathcal{S}_{p, t}} e^{R(T-t)^{2}}\left(\left|\varphi(t) \mathfrak{P}_{A}(u)(t, y)\right|^{2}+\left|\mathbf{c}(d t) \varphi^{\prime}(t) u(t, y)\right|^{2}\right) d y d t .
$$

Now we exploit our assumption

$$
\left|\mathfrak{P}_{A}(u)(x)\right| \leq P(u, x)|u(x)| \quad \text { for } x \in M
$$

about the perturbation with locally bounded $P(u, \cdot)$, say

$$
|P(u,(t, y))| \leq C_{0}:=\max _{x \in K}|P(u, x)| \quad \text { for all } y \in \mathcal{S}_{p, t}, t \in[0, T]
$$

where $K$ is a suitable compact set. We obtain at once

$$
\begin{aligned}
\left(1-\frac{2 C C_{0}}{R}\right) I & \leq \frac{2 C}{R} \int_{t=0}^{T} \int_{\mathcal{S}_{p, t}} e^{R(T-t)^{2}}\left|\mathbf{c}(d t) \varphi^{\prime}(t) u(t, y)\right|^{2} d y d t \\
& \leq \frac{2 C}{R} e^{R T^{2} / 25} \int_{t=0}^{T} \int_{\mathcal{S}_{p, t}}\left|\mathbf{c}(d t) \varphi^{\prime}(t) u(t, y)\right|^{2} d y d t .
\end{aligned}
$$

Here we use that $\varphi^{\prime}(t)=0$ for $0 \leq t \leq 0.8 T$ so that we can estimate the exponential and pull it in front of the integral. Returning to (2.6) yields

$$
\int_{0}^{\frac{T}{2}} \int_{S_{t}}\|u(t, y)\|^{2} d y d t \leq \frac{R}{R-2 C C_{0}} \frac{2 C}{R} e^{-21 R T^{2} / 100} \int_{0}^{T} \int_{\mathcal{S}_{p, t}}\left|\mathbf{c}(d t) \varphi^{\prime}(t) u(t, y)\right|^{2} d y d t
$$

which gives the result as $R \rightarrow \infty$. 
Remark 2.8. The preceding proof consists only of a short modification of the usual way one obtains weak UCP for the unperturbed equation from the Carleman estimate. The point of our proof for the perturbed case is that the unmodified Carleman estimate for the unperturbed operator suffices (Lemma 2.4 a). In the Appendix to this Note we give an alternative proof of Theorem 2.7 by modifying Lemma 2.4 and establishing a new Carleman inequality.

2.4. Application to the linearization of Seiberg-Witten equations. As an immediate application of Theorem 2.7, we show that, at any solution to the SeibergWitten equations, the linearization operator also enjoys the weak UCP. We only give an account of such linearization in the 3-dimensional case, since the corresponding claim in the 4-dimensional case can be established by a similar argument.

Let $Y$ be a closed, oriented 3-manifold equipped with a Riemannian metric $g$ and a Spin $^{\mathrm{c}}$ structure. A $\operatorname{Spin}^{\mathrm{c}}$ structure on $(Y, g)$ is a lift of the $S O(3)$-frame bundle on $Y$ to a $\operatorname{Spin}^{\mathrm{c}}(3)$-bundle $P$. Note that $\operatorname{Spin}^{\mathrm{c}}(3)=U(2)$. The determinant homomorphism $\operatorname{Spin}^{\mathrm{c}}(3) \rightarrow U(1)$ determines a principal $U(1)$-bundle, whose corresponding complex line bundle is called the determinant line bundle of the $\operatorname{Spin}^{\mathrm{c}}$ structure $P$.

Given a $\operatorname{Spin}^{\mathrm{c}}$ structure $P$, there is an associated $\operatorname{Spin}^{\mathrm{c}}$ bundle

$$
W=P \times_{U(2)} \mathbb{C}^{2},
$$

which is a complex vector bundle and a module over the bundle of Clifford algebras on $(Y, g)$. The bundle of Clifford algebras on $(Y, g)$ can be identified with the bundle of exterior algebras on $T^{*} Y$, but with a different algebra structure: the Clifford relation.

With the Levi-Civita connection $\nabla$ on the cotangent bundle $T^{*} Y$, a $U(1)$-connection $A$ on the determinant bundle $\operatorname{det}(W)$ determines a connection $\nabla_{A}$ on $W$ such that, for $v$ and $\psi$ sections of $T^{*} Y$ and $W$ respectively, $\nabla_{A}$ satisfies

$$
\nabla_{A}(\mathbf{c}(v) \psi)=\mathbf{c}(\nabla v) \psi+\mathbf{c}(v) \nabla_{A}(\psi)
$$

Then $\nabla_{A}$ is called a $\operatorname{Spin}^{\mathrm{c}}$ connection on $W$. Applying the Clifford multiplication, we can define a Dirac operator $\not_{A}=\mathbf{c} \circ \nabla_{A}: \Gamma(W) \rightarrow \Gamma(W)$.

The Seiberg-Witten equations on $(Y, g)$, for a pair $(A, \psi)$ consisting of a $U(1)$ connection $A$ on the determinant line bundle of $\mathfrak{s}$ and a spinor section $\psi$ of $W$, is given by 12] [10] [14]:

$$
\left\{\begin{array}{l}
\not_{A} \psi=0 \\
* F_{A}=\sigma(\psi, \psi)
\end{array}\right.
$$

where $\sigma(\psi, \psi)$ is an $\mathbb{R}$-bilinear form on sections of spinor bundle, an imaginary valued 1-form on $Y$ given by

$$
\sigma(\psi, \psi)=\frac{i}{2} \Im\left(\left\langle\mathbf{c}\left(e_{i}\right) \psi, \psi\right\rangle\right) e^{i} \in \Omega^{1}(Y, i \mathbb{R}) .
$$

Note that these equations are gauge invariant under the action of gauge group $\mathcal{G}=$ $\operatorname{Map}(Y, U(1)): u(A, \psi)=\left(A-2 u^{-1} d u, u \psi\right)$ for any $u \in \mathcal{G}=\operatorname{Map}(Y, U(1))$. 
Let $(A, \psi)$ be a smooth solution to the Seiberg-Witten equations (2.9), then the spinor $\psi$ satisfies

$$
|\psi|^{2} \leq \max _{y \in Y}\{0,-s(y)\}
$$

where $s(y)$ is the scalar curvature for $(Y, g)$ [12].

The linearization of the Seiberg-Witten equations (2.9) at $(A, \psi)$, together with the linearization of the gauge action, gives rise to the following linear elliptic system of equations for $(\alpha, \phi)$ (a pair of an imaginary valued 1-form $\alpha$ and a spinor section):

$$
\left\{\begin{array}{l}
d^{*} a+i \Im\langle\psi, \phi\rangle=0, \\
* d \alpha-\sigma(\psi, \phi), \\
\not_{A} \phi+\frac{1}{2} \mathbf{c}(\alpha) \psi=0 .
\end{array}\right.
$$

Then we have the following weak unique continuation result for the linearization (2.11) of the Seiberg-Witten equations, whose proof follows from Theorem 2.7 and the pointwise bound in 2.10 .

Corollary 2.9. If $(A, \psi)$ is a solution of the Seiberg-Witten equations (2.9), then as a solution to (2.11), $(\alpha, \phi)$ satisfies the weak unique continuation property.

\section{Perturbations of the Chern-Simons-Dirac functional}

We consider perturbations of the Chern-Simons-Dirac functional of the form proposed in [5] and [11]. The setup is as in the last paragraph: we have a closed compact connected oriented smooth 3 -manifold $Y$, with a fixed Spin ${ }^{\mathrm{c}}$-structure $\mathfrak{s}$, and with a choice of a Riemannian metric. We consider the configuration space $\mathcal{A}$ of pairs $(A, \psi)$ of a $U(1)$-connection and a spinor section, with the action of the gauge group $\mathcal{G}$ as above. The spaces $\mathcal{A}$ and $\operatorname{Lie}(\mathcal{G})$ are completed in suitable Sobolev norms (see e.g. [14]). Moreover, on the non-compact 4 -manifold $Y \times \mathbb{R}$ we consider the Spin ${ }^{\mathrm{c}}$-structure obtained as pullback of $\mathfrak{s}$ on $Y$, and the cylindrical metric. The configuration space is given by the set of finite energy pairs $(\mathbb{A}, \Psi)$ of a $U(1)$-connection and a spinor on $Y \times \mathbb{R}$, acted upon by the corresponding gauge group. The finite energy condition consists of the property that, after a gauge transformation that kills the $d t$ component of $\mathbb{A}$, the resulting $(A(t), \psi(t))$ has time derivative in $L^{2}$. In this case the configuration space can be topologized by suitable weighted Sobolev norms. Since we do not need the details here, we refer to [14].

3.1. Case I. We consider the space of functions

$$
\bigcup_{N \geq b_{1}, K>0} \mathrm{C}^{\infty}\left(\mathbb{R}^{N}, \mathbb{R}\right) \times \mathrm{C}^{\infty}\left(\mathbb{R}^{K}, \mathbb{R}\right),
$$

where $b_{1}$ is the first Betti number of the 3-manifold $Y$. 
In order to have the correct setup for transversality arguments, we need a Banach space of parameters, hence we select as perturbation parameter space $\mathcal{P}$ a subspace of (3.1) of functions $\left(\mathfrak{p}_{1}, \mathfrak{p}_{2}\right)$ with finite Floer $\epsilon$-norm.

The Floer $\epsilon$-norm of $\left(\mathfrak{p}_{1}, \mathfrak{p}_{2}\right)$ is defined as follows: choose $\underline{\epsilon}=\left(\epsilon_{k}\right)_{k \in \mathbb{N}}$ to be a given sequence of positive real numbers, and set

$$
\left\|\left(\mathfrak{p}_{1}, \mathfrak{p}_{2}\right)\right\|_{\epsilon}=\sum_{k \geq 0}\left(\epsilon_{k} \sup \left|\nabla^{k} \mathfrak{p}_{1}\right|+\epsilon_{k} \sup \left|\nabla^{k} \mathfrak{p}_{2}\right|\right)
$$

Following the argument of Lemma 5.1 of [9], the sequence $\underline{\epsilon}$ can be chosen such that $\mathcal{P}$ is a Banach space and $\mathcal{P} \cap L_{k}^{2}$ is dense in $L_{k}^{2}$. We require that $\mathfrak{p}_{1}$ is invariant under the actions of $H^{1}(Y, Z)$ on $\mathbb{R}^{b_{1}}$.

3.1.1. For any 3-manifold $Y$, endowed with a Riemannian metric $g$ and a fixed $\operatorname{Spin}^{\mathrm{c}}$ structure $\mathfrak{s}$, we choose a complete $L^{2}$-basis $\left\{\nu_{j}\right\}_{j=1}^{\infty}$ of imaginary-valued 1 -forms on $Y$. We also choose a complete $L^{2}$-basis $\left\{\mu_{j}\right\}_{j=1}^{\infty}$ for the co-closed (imaginary-valued) 1-forms on $Y$. Under the Hodge decomposition, we have

$$
\Lambda_{L^{2}}^{1}(Y, i \mathbb{R})=H^{1}(Y, i \mathbb{R}) \oplus \operatorname{Im}\left(d^{*}\right) \oplus \operatorname{Im}(d),
$$

and the $\left\{\mu_{j}\right\}_{j=1}^{\infty}$ span the space $H^{1}(Y, i \mathbb{R}) \oplus \operatorname{Im}\left(d^{*}\right)$.

3.1.2. Fix a $U(1)$-connection $A_{0}$ on the determinant bundle $\operatorname{det}(\mathfrak{s})$. To each co-closed 1 -form $\mu_{j}$ we associate a function on the configuration space $\mathcal{A}$, defined as

$$
\tau_{j}(A, \psi)=\int_{Y}\left(A-A_{0}\right) \wedge * \mu_{j} .
$$

For simplicity, we assume that the $\left\{\mu_{j}\right\}_{j=1}^{b_{1}}$ form a basis of $H^{1}(Y, i \mathbb{R})$, and $\left[* \mu_{j}\right]=0$ for $j>b_{1}$. It is easy to see that the following properties are satisfied:

(1) $\tau_{j}$ is invariant under gauge transformations for $j>b_{1}$;

(2) the map

$$
\left(\tau_{1}, \cdots, \tau_{b_{1}}\right): \mathcal{A} \rightarrow \mathbb{R}^{b_{1}}
$$

is equivariant with respect to the action of gauge transformations $\lambda: Y \rightarrow U(1)$ on $\mathcal{A}$ and the action of the corresponding $[\lambda] \in H^{1}(Y, \mathbb{Z}) \cong \mathbb{Z}^{b_{1}}$ on $\mathbb{R}^{b_{1}}$ as a translation by

$$
\left(\left[\lambda^{-1} d \lambda\right] \cup\left[* \mu_{1}\right],\left[\lambda^{-1} d \lambda\right] \cup\left[* \mu_{2}\right], \cdots,\left[\lambda^{-1} d \lambda\right] \cup\left[* \mu_{b_{1}}\right]\right) \cap[Y] .
$$

3.1.3. To each imaginary-valued 1 -form $\nu_{j}$, we associate a function $\zeta_{j}$ on $\mathcal{A}$, defined as the quadratic form in the spinor $\psi$,

$$
\zeta_{j}(\psi, \psi)=\int_{Y}\left\langle\mathbf{c}\left(\nu_{j}\right) \psi, \psi\right\rangle
$$

where $\langle\cdot, \cdot\rangle$ is the Hermitian metric on the space of spinors. It is easy to see that $\zeta_{j}$ is gauge invariant and real-valued. 
3.1.4. Now we choose any function $\mathfrak{p}_{1} \in \mathrm{C}^{\infty}\left(\mathbb{R}^{N}, \mathbb{R}\right)$ and $\mathfrak{p}_{2} \in \mathrm{C}^{\infty}\left(\mathbb{R}^{K}, \mathbb{R}\right)$ (for $N \geq b_{1}$, $K>0)$ where $\mathfrak{p}_{1}$ is invariant under the action of $H^{1}(Y, \mathbb{Z})$ on $\mathbb{R}^{b_{1}} \subset \mathbb{R}^{N}$. We can define a function on $\mathcal{A} / \mathcal{G}$ as

$$
\mathfrak{p}_{1}\left(\tau_{1}, \ldots, \tau_{N}\right)+\mathfrak{p}_{2}\left(\zeta_{1}, \ldots, \zeta_{K}\right) .
$$

Let $C S D$ be the Chern-Simons-Dirac functional

$$
C S D(A, \psi)=-\frac{1}{2} \int_{Y}\left(A-A_{0}\right) \wedge\left(F_{A}+F_{A_{0}}\right)+\int_{Y}\left\langle\psi, \not_{A} \psi\right\rangle,
$$

where $\not_{A}$ is the self adjoint Dirac operator on the compact 3-manifold $Y$ twisted with the $U(1)$-connection $A$. We perturb this functional as

$$
\widetilde{C S D}(A, \psi)=C S D(A, \psi)+\mathfrak{p}_{1}\left(\tau_{1}, \ldots, \tau_{N}\right)+\mathfrak{p}_{2}\left(\zeta_{1}, \ldots, \zeta_{K}\right) .
$$

3.1.5. For each $\left(\mathfrak{p}_{1}, \mathfrak{p}_{2}\right) \in \mathcal{P}$, the gradient of (3.3), with respect to the $L^{2}$-metric on $\mathcal{A} / \mathcal{G}$, is computed in the following lemma [5].

Lemma 3.1. Consider the perturbed functional (3.3) with

$$
\left(\mathfrak{p}_{1}, \mathfrak{p}_{2}\right) \in \mathcal{P} \cap\left(\mathrm{C}^{\infty}\left(\mathbb{R}^{N}, \mathbb{R}\right) \times \mathrm{C}^{\infty}\left(\mathbb{R}^{K}, \mathbb{R}\right)\right) .
$$

The $L^{2}$-gradient of (3.3) at $(A, \psi)$ is given by

$$
\nabla \widetilde{C S D}(A, \psi)=\left\{\begin{array}{l}
* F_{A}-\sigma(\psi, \psi)-\sum_{j=1}^{N} \frac{\partial \mathfrak{p}_{1}}{\partial \tau_{j}} \mu_{j} \\
\not_{A} \psi-\sum_{j=1}^{K} \frac{\partial \mathfrak{p}_{2}}{\partial \zeta_{j}} \mathbf{c}\left(\nu_{j}\right) \psi
\end{array}\right.
$$

3.1.6. There are two types of perturbed Dirac equations that we obtain from Lemma 3.1. In fact, the critical points equations for the functional (3.3) provide perturbed 3-dimensional Seiberg-Witten equations of the form

$$
\left\{\begin{array}{l}
* F_{A}=\sigma(\psi, \psi)+\sum_{j=1}^{N} \frac{\partial \mathfrak{p}_{1}}{\partial \tau_{j}} \mu_{j} \\
\not_{A} \psi=\sum_{j=1}^{K} \frac{\partial \mathfrak{p}_{2}}{\partial \zeta_{j}} \mathbf{c}\left(\nu_{j}\right) \psi
\end{array}\right.
$$

where $\sigma(\psi, \psi)$ is a quadratic form in the spinor given by $\sum_{i}\left\langle\mathbf{c}\left(e_{i}\right) \psi, \psi\right\rangle e^{i}$, in dual local basis $\left\{e_{i}\right\}$ and $\left\{e^{i}\right\}$ of $T Y$ and $T^{*} Y$. Similarly, the gradient flow equations for the functional (3.3) correspond to perturbed 4-dimensional Seiberg-Witten equations (in 
a temporal gauge) of the form

$$
\left\{\begin{array}{l}
\frac{d A(t)}{d t}=-* F_{A(t)}+\sigma(\psi(t), \psi(t))+\sum_{j=1}^{N} \frac{\partial \mathfrak{p}_{1}}{\partial \tau_{j}} \mu_{j} \\
\frac{d \psi(t)}{d t}=-\not_{A(t)} \psi(t)+\sum_{j=1}^{K} \frac{\partial \mathfrak{p}_{2}}{\partial \zeta_{j}} \mathbf{c}\left(\nu_{j}\right) \psi(t) .
\end{array}\right.
$$

3.1.7. We can prove the following weak unique continuation result for solutions of the perturbed Seiberg-Witten equations (3.5) and (3.6).

Theorem 3.2. If $(A, \psi)$ is a solution of (3.5), and the spinor $\psi$ vanishes on a nonempty open set, then $\psi$ vanishes everywhere on the compact connected 3-manifold $Y$. Suppose then that $(\mathbb{A}, \Psi)$ is a pair of connection and spinor on the non-compact fourdimensional manifold $Y \times \mathbb{R}$, with cylindrical metric and spinor bundle $S=S^{+} \oplus S^{-}$ obtained by pulling back the $\operatorname{Spin}^{\mathrm{c}}$-structure $\mathfrak{s}$ on $Y$. If $(\mathbb{A}, \Psi)$ is gauge equivalent to $(A(t), \psi(t))$ in a temporal gauge, satisfying (3.6), and $\Psi$ vanishes on a non-empty open set, then $\Psi$ vanishes everywhere on $Y \times \mathbb{R}$.

Proof. The result follows in both cases directly from Theorem 2.7, with the non-linear and global perturbation

$$
\mathfrak{P}_{A}(\psi):=-\sum_{j=1}^{K} \frac{\partial \mathfrak{p}_{2}}{\partial \zeta_{j}} \mathbf{c}\left(\nu_{j}\right) \psi,
$$

so that the Dirac equation in (3.5) becomes of the form $\widetilde{\phi}_{A} \psi=0$, with

$$
\widetilde{\not}_{A} \psi=\not_{A} \psi+\mathfrak{P}_{A}(\psi) \text {. }
$$

The Dirac equation in (3.6) can also be written as $\widetilde{D_{\mathrm{A}}} \Psi=0$, with the perturbation term $\mathfrak{P}_{A}(\Psi)$ which only differs from (3.7) by a unitary operator $\mathbf{c}(d t)$ and

$$
\widetilde{D_{\mathrm{A}}} \Psi=\not D_{\mathrm{A}} \Psi-\mathfrak{P}_{A}(\Psi),
$$

with $\not D_{\mathrm{A}}$ the Dirac operator on $Y \times \mathbb{R}$ and $\mathfrak{P}_{A}(\Psi)=\sum_{j=1}^{K} \frac{\partial \mathfrak{p}_{2}}{\partial \zeta_{j}} \mathbf{c}\left(\nu_{j}\right) \Psi$,

$$
\not D_{\mathrm{A}}: \Gamma\left(Y \times \mathbb{R}, S^{+}\right) \rightarrow \Gamma\left(Y \times \mathbb{R}, S^{-}\right) \text {. }
$$

3.2. Case II. Here we consider a version of the perturbations introduced in [11], where, in addition to the perturbations $\mathfrak{p}_{1}\left(\tau_{1}, \ldots, \tau_{N}\right)$ and $\mathfrak{p}_{2}\left(\zeta_{1}, \ldots, \zeta_{K}\right)$ defined as in $\S 3.1 .2$ and 3.1.4, we introduce further perturbations depending on both connection and spinor, described as follows. 
3.2.1. Let $G$ be the Green operator for the ordinary Laplacian on $Y$. If $\mathcal{G}_{e}$ is the identity component of the gauge group $\mathcal{G}$, we can consider the subgroup $\mathcal{H} \subset \mathcal{G}_{e}$

$$
\mathcal{H}=\left\{\lambda=e^{i f}, f: Y \rightarrow \mathbb{R} \text { such that } \int_{Y} f=0\right\},
$$

with $\mathcal{G} / \mathcal{H} \cong U(1) \times H^{1}(Y, \mathbb{Z})$. Consider also a fixed family of locally bounded spinors $\left\{\psi_{i}\right\}_{i=1,2, \ldots}$ on $Y$. We choose the family $\left\{\psi_{i}\right\}_{i=1,2, \ldots}$ so that they form a complete $L^{2}$-basis of the space of spinor sections, and they are eigenvectors of the fixed Dirac operator $\not_{A_{0}}$, where $A_{0}$ is the fixed $U(1)$-connection that appears in the definition of Chern-Simons-Dirac functional (3.2). We set

$$
\eta_{i}(A, \psi):=\int\left\langle e^{-G d^{*}\left(A-A_{0}\right)} \psi_{i}, \psi\right\rangle
$$

The following properties are satisfied:

(1) $\eta_{i}$ is invariant under $\mathcal{H}$;

(2) the map

$$
\left(\eta_{1}, \ldots, \eta_{L}\right): \mathcal{A} \rightarrow \mathbb{C}^{L}
$$

is equivariant with respect to the action of $\mathcal{G}$ on $\mathcal{A}$ and the corresponding action of $U(1) \times H^{1}(Y, \mathbb{Z})$ on $\mathbb{C}^{L}$.

3.2.2. We choose a class of functions $\mathfrak{p}_{3}: \mathbb{C}^{L} \rightarrow \mathbb{R}$ which is invariant with respect to the action of $U(1) \times H^{1}(Y, \mathbb{Z})$. We consider the corresponding perturbation term

$$
\mathfrak{p}_{3}\left(\eta_{1}, \ldots, \eta_{L}\right): \mathcal{A} \rightarrow \mathbb{R}
$$

and the resulting perturbed Chern-Simons-Dirac functional of the form

$$
\widetilde{C S D}(A, \psi)=C S D(A, \psi)+\mathfrak{p}_{1}\left(\tau_{1}, \ldots, \tau_{N}\right)+\mathfrak{p}_{2}\left(\zeta_{1}, \ldots, \zeta_{K}\right)+\mathfrak{p}_{3}\left(\eta_{1}, \ldots, \eta_{L}\right) .
$$

Lemma 3.3. The $L^{2}$-gradient of the perturbed functional (3.9), with the additional perturbation $\mathfrak{p}_{3}$, is given by

$$
\nabla \widetilde{C S D}(A, \psi)=\left\{\begin{array}{l}
* F_{A}-\sigma(\psi, \psi)-\sum_{j=1}^{N} \frac{\partial \mathfrak{p}_{1}}{\partial \tau_{j}} \mu_{j}-\sum_{\ell=1}^{L} \frac{\partial \mathfrak{p}_{3}}{\partial \eta_{\ell}} d G\left(i \Im\left\langle e^{-G d^{*}\left(A-A_{0}\right)} \psi_{\ell}, \psi\right\rangle\right) \\
\not_{A} \psi-\sum_{i=1}^{K} \frac{\partial \mathfrak{p}_{2}}{\partial \zeta_{i}} \mathbf{c}\left(\nu_{i}\right) \psi-\sum_{\ell=1}^{L} \frac{\partial \mathfrak{p}_{3}}{\partial \eta_{\ell}} e^{-G d^{*}\left(A-A_{0}\right)} \psi_{\ell}
\end{array}\right.
$$

Thus, the Dirac equations derived from the perturbed critical point equations

$$
\nabla \widetilde{C S D}(A, \psi)=0
$$

and perturbed flow lines equations

$$
\frac{d}{d t}(A(t), \psi(t))+\nabla \widetilde{C S D}(A(t), \psi(t))=0
$$


for the functional (3.9) are, respectively, of the form

$$
\not_{A} \psi-\sum_{i=1}^{K} \frac{\partial \mathfrak{p}_{2}}{\partial \zeta_{i}} \mathbf{c}\left(\nu_{i}\right) \psi-\sum_{\ell=1}^{L} \frac{\partial \mathfrak{p}_{3}}{\partial \eta_{\ell}} e^{-G d^{*}\left(A-A_{0}\right)} \psi_{\ell}=0
$$

on the compact 3-manifold $Y$ and, on the non-compact four-manifold $Y \times \mathbb{R}$,

$$
\left(\frac{\partial}{\partial t}+\not_{A(t)}\right) \psi(t)-\sum_{i=1}^{K} \frac{\partial \mathfrak{p}_{2}}{\partial \zeta_{i}} \mathbf{c}\left(\nu_{i}\right) \psi(t)-\sum_{\ell=1}^{L} \frac{\partial \mathfrak{p}_{3}}{\partial \eta_{\ell}} e^{-G d^{*}\left(A(t)-A_{0}\right)} \psi_{\ell}=0
$$

3.3. The UCP problem for Case II. Unlike the perturbations $\mathfrak{p}_{1}$ and $\mathfrak{p}_{2}$ of Case I, the problem of UCP is a lot more delicate for the perturbations $\mathfrak{p}_{3}$ of Case II.

We begin with a very simple example, again taken from the theory of ordinary differential equations, which shows that UCP may fail for a toy model of the perturbations of Case II. More precisely, we consider perturbations of the form

$$
\left.\mathfrak{P}_{a}(u)\right|_{x}:=\langle u, a\rangle a(x),
$$

for a fixed $L^{2}$-spinor $a(x)$, and with $\langle\cdot, \cdot\rangle$ the $L^{2}$-inner product on spinors,

$$
\langle u, a\rangle=\int_{Y}\langle u(x), a(x)\rangle d v(x) .
$$

We take this class as a simplified version of (3.8), and of the resulting $\mathfrak{p}_{3}$. In any case, we are only looking at the Dirac part of the Seiberg-Witten equations.

Example 3.4. Let $a:[0,2] \rightarrow \mathbb{R}$ be a continuous function which vanishes for $x \in[0,1]$ and satisfies

$$
\int_{1}^{2} a(s) d s=\sqrt{2}
$$

Then the function

$$
u(x):= \begin{cases}0 & \text { for } x \in[0,1) \\ \int_{1}^{x} a(s) d s & \text { for } x \in[1,2]\end{cases}
$$

belongs to $C^{1}([0,2])$ and satisfies

$$
u^{\prime}(x)=\langle u, a\rangle a(x) \quad(\text { for } x \in[0,2]),
$$

since

$$
\langle u, a\rangle=\int_{0}^{2} u(t) a(t) d t=\frac{1}{2} u^{2}(2)=1
$$


This shows that, already in the one-dimensional case and with the simplified perturbations of the form (3.13), weak UCP fails. This means that, in general, it will be difficult to expect (even weak) UCP for the perturbations of Case II.

However, there are special conditions under which perturbations of the form (3.13) do still satisfy weak UCP:

Lemma 3.5. Assume that one of the following conditions is satisfied:

- the spinor a does not vanish on any open subset, or

- The support of a is contained in the interior of the support of the solution u under consideration, or, alternatively put, there exists a positive constant $C_{0}$ such that $|a(x)| \leq C_{0}|u(x)|$ for all $x \in M$.

Then the Dirac equation with a perturbation of the form (3.13) satisfies the weak UCP.

Proof. The second listed condition makes our perturbation admissible in the sense of Definition 2.5 (cf. the second item of Example 2.6), and weak UCP follows from Theorem 2.7.

Now we deal with the first listed condition. Let $u$ be a solution of the perturbed equation

$$
\not \not_{A} u(x)+\langle u, a\rangle a(x)=0, \quad x \in M .
$$

If $\Omega$ is open in $M$ and $u$ vanishes identically on $\Omega$, then either $\langle u, a\rangle$ must vanish or $a$ must vanish identically on $\Omega$. In the first case we have no longer a perturbation and we are left with Lemma 2.3 which guarantees weak UCP.

The second case is excluded by the first listed condition of our lemma.

By the choice of the $\left\{\psi_{i}\right\}_{i=1,2, \cdots}$, we know that each $\psi_{i}$, as an eigenvector of the Dirac operator $\not_{A_{0}}$, satisfies the weak UCP by the argument of Remark 2.3, so the additional perturbation in (3.11) and (3.12) satisfies the first condition of Lemma 3.5. The argument given in the proof of Lemma 3.5 for perturbations of the form (3.13) extends easily to the more general case of perturbed equations of the form (3.11) and (3.12).

Thus, we have proved the following weak unique continuation result for solutions of the perturbed Seiberg-Witten equations (3.11) and (3.12).

Theorem 3.6. If $(A, \psi)$ is a solution of (3.11), and the spinor $\psi$ vanishes on an open set, then $\psi$ vanishes everywhere on the compact connected 3-manifold $Y$. Suppose then that $(\mathbb{A}, \Psi)$ is pair of a connection and spinor on the non-compact four-dimensional manifold $Y \times \mathbb{R}$, with cylindrical metric and spinor bundle $S=S^{+} \oplus S^{-}$obtained by pulling back the $\operatorname{Spin}^{\mathrm{c}}$-structure $\mathfrak{s}$ on $Y$. If $(\mathbb{A}, \Psi)$ is gauge equivalent to $(A(t), \psi(t))$ in a temporal gauge, satisfying (3.12), and $\Psi$ vanishes on an open set, then $\Psi$ vanishes everywhere on $Y \times \mathbb{R}$. 
3.4. Linearizations. A detailed discussion of the transversality results for monopole moduli spaces resulting from these perturbed equations will be presented elsewhere. However, in view of such application, we state another result, which follows from Theorem 2.7. Recall that, in the proof of transversality for monopole equations (see [14, §2.3]), one argues that a possible element in the Cokernel of the linearization must in fact vanish identically. By varying the choice of the perturbation (transversality of the universal moduli space), the question can be reduced to an argument involving weak UCP for the adjoint of the (perturbed) Dirac operator (we refer to [14, §2.3] for details).

There is a conjecture by Laurent Schwartz [16] that weak UCP for an elliptic differential operator will always imply weak UCP for its formal adjoint. The matter was discussed in [4] in detail, but is still unsolved, see also [3, Section 2.2]. However, in the special case considered here we can prove similar results for the linearizations and their adjoints, applying Theorem 2.7.

Consider the linearization of equations (3.5) and (3.6). This gives linear operators $\mathfrak{d}_{(A, \psi)}$ and $\mathfrak{D}_{(\mathbb{A}, \Psi)}$ respectively. Notice that the linearization $\mathfrak{d}_{(A, \psi)}$ or its adjoint $\mathfrak{d}_{(A, \psi)}^{*}$ acts on pairs of a 1 -form and spinor $(\alpha, \phi)$, and it involves a term of the form $\alpha \cdot \psi$. Thus, this operator is not of the type directly considered in Theorem 2.7, hence we shall not apply directly the result to this operator. However, for the proof of transversality (see e.g. [14]) it is sufficient to know that the operators $\mathfrak{d}_{(A, \psi)}^{*}$ and $\mathfrak{D}_{(\mathbb{A}, \Psi)}^{*}$ acting on "pure spinor elements" $(0, \phi)$ satisfy the UCP property, and this can be shown using Theorem 2.7. The case of $\mathfrak{D}_{(\mathbb{A}, \Psi)}$ is completely analogous.

Corollary 3.7. Let the linear operators $\mathfrak{d}_{(A, \psi)}^{*}$ and $\mathfrak{D}_{(\mathbb{A}, \Psi)}^{*}$ be the $L^{2}$-adjoint to the linearizations of equation (3.5) and equation (3.6), respectively. If an element $(0, \phi)$ of pure spinor part is in the kernel of $\mathfrak{d}_{(A, \psi)}^{*}$ or $\mathfrak{D}_{(\mathbb{A}, \Psi)}^{*}$, and the spinor $\phi$ vanishes on a non-empty open set, then $\phi$ vanishes identically on $Y($ or $Y \times \mathbb{R})$.

Proof. The adjoint operators $\not_{A}^{*}$ and $\not_{\mathrm{A}}{ }^{*}$ are again of Dirac type. In particular the adjoint operators $\mathfrak{d}_{(A, \psi)}^{*}$ and $\mathfrak{D}_{(\mathbb{A}, \Psi)}^{*}$, acting on elements of pure spinor part, are Diractype operators with admissible perturbations. Then the weak UCP for an element in the kernel of $\mathfrak{d}_{(A, \psi)}^{*}$ and $\mathfrak{D}_{(\mathbb{A}, \Psi)}^{*}$ follows from Theorem 2.7.

\section{APPEndiX}

In this Appendix we give an alternative proof of Theorem 2.7 which is also instructive, though longer than the one we have given in the paper.

We prove that the perturbed Carleman inequality

$$
R \int_{t=0}^{T} \int_{\mathcal{S}_{p, t}} e^{R(T-t)^{2}}|v(t, y)|^{2} d y d t \leq C \int_{t=0}^{T} \int_{\mathcal{S}_{p, t}} e^{R(T-t)^{2}}\left|\widetilde{\not_{\mathrm{A}}} v(t, y)\right|^{2} d y d t
$$


holds for any real $R$ sufficiently large.

In order to make the various steps of the proof more transparent, we have subdivided the proof in short paragraphs.

4.0.1. First consider a few technical points. The Dirac operator $\not_{\mathrm{A}}$ has the form $G(t)\left(\partial_{t}+\mathcal{B}_{t}\right)$ on the annular region $\left\{\mathcal{S}_{p, t}\right\}_{t \in[0, T]}$, and it is obvious that we have wUCP for the operator $\partial_{t}+\mathcal{B}_{t}$ if and only if we have it for $\not_{\mathrm{A}}$. Moreover, we have by Lemma 2.2 that $\mathcal{B}_{t}=B_{t}+C_{t}$ with a self-adjoint elliptic differential operator $B_{t}$ and an antisymmetric operator $C_{t}$ of order zero, both on $\mathcal{S}_{p, t}$.

4.0.2. Now make the substitution $v=: e^{-R(T-t)^{2} / 2} v_{0}$ which replaces (4.1) by

$$
\begin{aligned}
& R \int_{0}^{T} \int_{\mathcal{S}_{t}}\left|v_{0}(t, y)\right|^{2} d y d t \\
& \leq C \int_{0}^{T} \int_{\mathcal{S}_{t}}\left|\frac{\partial v_{0}}{\partial t}+\mathcal{B}_{t} v_{0}+R(T-t) v_{0}+e^{\frac{R}{2}(T-t)^{2}} \mathfrak{P}_{A}(v)\right|^{2} d y d t .
\end{aligned}
$$

We shall denote the integral on the left hand side by $J_{0}$ and the integral on the right hand side by $J_{1}$.

4.0.3. Now we prove (4.2). Decompose $\frac{\partial}{\partial t}+\mathcal{B}_{t}+R(T-t)+\mathfrak{P}_{A}$ into its symmetric part $B_{t}+R(T-t)+\mathfrak{P}_{A}$ and anti-symmetric part $\partial_{t}+C_{t}$. This gives

$$
\begin{aligned}
J_{1}= & \iint\left|\left(\frac{\partial v_{0}}{\partial t}+C_{t} v_{0}\right)+\left(B_{t} v_{0}+R(T-t) v_{0}+e^{\frac{R}{2}(T-t)^{2}} \mathfrak{P}_{A}(v)\right)\right|^{2} d y d t \\
= & \underbrace{\iint\left|\frac{\partial v_{0}}{\partial t}+C_{t} v_{0}\right|^{2} d y d t}_{J_{\text {skew }}}+\underbrace{\iint \mid\left(B_{t}+R(T-t)\right) v_{0}} \\
& +\left.e^{\frac{R}{2}(T-t)^{2}} \mathfrak{P}_{A}(v)\right|^{2} d y d t \\
& +2 \Re \iint\left\langle\frac{\partial v_{0}}{\partial t}+C_{t} v_{0}, B_{t} v_{0}+R(T-t) v_{0}\right\rangle+e^{\frac{R}{2}(T-t)^{2}} \mathfrak{P}_{A}(v) d y d t \\
= & : J_{\text {skew }}+J_{\text {sym }}+J_{\text {mix }} .
\end{aligned}
$$

4.0.4. First we investigate the term $J_{\text {mix }}$. We integrate by parts and use the identities for the real part

$$
\Re\langle f, P f\rangle=\frac{1}{2}\left\langle f,\left(P+P^{*}\right) f\right\rangle,
$$

and

$$
2 \Re\langle a, b\rangle \geq-2|\langle a, b\rangle| \geq-\left(\epsilon|b|^{2}+\frac{|a|^{2}}{\epsilon}\right) .
$$


We get, almost like in [3, p.30],

$$
J_{\text {mix }}=R J_{0}+J_{3}+J_{\text {skew.pert }}
$$

4.0.5. The first term on the right in (4.3) is

$$
R J_{0}=R \int_{0}^{T}\left|v_{0}\right|^{2} d t
$$

The second term on the right in (4.3) is the most delicate and was thoroughly analyzed in the unperturbed case:

$$
J_{3}:=\iint\left\langle v_{0},-\frac{\partial B_{t}}{\partial t} v_{0}+\left[B_{t}, C_{t}\right] v_{0}\right\rangle d y d t .
$$

The third term on the right in (4.3) is

$$
\begin{aligned}
J_{\text {skew.pert }}- & =2 \Re \iint\left\langle\frac{\partial v_{0}}{\partial t}+C_{t} v_{0}, e^{\frac{R}{2}(T-t)^{2}} \mathfrak{P}_{A}(v)\right\rangle d y d t \\
\geq & -\iint\left|\frac{\partial v_{0}}{\partial t}+C_{t} v_{0}\right|^{2} d y d t-\iint\left|e^{\frac{R}{2}(T-t)^{2}} \mathfrak{P}_{A}(v)\right|^{2} d y d t
\end{aligned}
$$

4.0.6. As a matter of fact the term $J_{\text {skew }}$ was not used at all for establishing the Carleman inequality in the unperturbed case. Hence we can balance the first negative term in the preceding estimate for $J_{\text {skew.pert }}$ with $J_{\text {skew }}$.

To balance the second negative term we decompose

$$
\begin{aligned}
J_{\text {sym }}=\epsilon \iint \mid\left(B_{t}+\right. & R(T-t))\left.v_{0}\right|^{2} d y d t \\
& +(1-\epsilon) \iint\left|\left(B_{t}+R(T-t)\right) v_{0}\right|^{2} d y d t \\
& +\iint\left|e^{\frac{R}{2}(T-t)^{2}} \mathfrak{P}_{A}(v)\right|^{2} d y d t+J_{\text {sym.pert }}
\end{aligned}
$$

with

$$
\begin{aligned}
J_{\text {sym.pert }}= & 2 \Re \iint\left\langle\left(B_{t}+R(T-t)\right) v_{0}, e^{\frac{R}{2}(T-t)^{2}} \mathfrak{P}_{A}(v)\right\rangle d y d t \\
\geq & -\epsilon \iint\left|\left(B_{t}+R(T-t)\right) v_{0}\right|^{2} d y d t \\
& -\frac{1}{\epsilon} \iint\left|e^{\frac{R}{2}(T-t)^{2}} \mathfrak{P}_{A}(v)\right|^{2} d y d t .
\end{aligned}
$$


4.0.7. Now the second (negative) term in the estimate for $J_{\text {skew.pert }}$ balances exactly with the third term in the decomposition of $J_{\text {sym }}$. Moreover, the first (negative) term in the estimate for $J_{\text {sym.pert }}$ balances exactly with the first term in the decomposition of $J_{\text {sym }}$. Note, that a fraction of $\iint\left|\left(B_{t}+R(T-t)\right) v_{0}\right|^{2} d y d t$ (say, the second term in the decomposition of $J_{\text {sym }}$ ) was needed for balancing $J_{3}$ in the unperturbed case (see also Remark 4.1). So, we must choose $\epsilon$ sufficiently small, say $\epsilon=\frac{1}{4}$.

To balance the second (negative) term in the estimate for $J_{\text {sym.pert }}$ we are left with the only positive term not yet (fully) used: $R J_{0}$.

4.0.8. Let us first consider the case of admissible perturbations satisfying (2.5). Introducing the perturbation induces a final error

$$
J_{\text {err }}=\int_{0}^{T} \int_{\mathcal{S}_{p, t}}\left|v_{0}\right|^{2}\left(R-\frac{1}{\epsilon}|P(v)|^{2}\right) d y d t
$$

with fixed $\epsilon$. For fixed $v$ we can choose a positive $R_{0}$ such that the error term $J_{\text {err }}$ becomes $\geq 0$ for all $R \geq R_{0}$.

Remark 4.1. In the unperturbed case, the completely elementary character of the arguments was made possible by exploiting one single non-elementary ingredient, Gårding's inequality (here the equivalence of the first Sobolev norm to the graph norm of any linear first order elliptic differential operator). It was used for estimating the term $J_{3}$ by $\frac{1}{2} J_{\text {sym }}$ and a fraction of $J_{0}$. For the perturbed case, it is worth mentioning that we do not need any kind of non-linear elliptic estimate but can keep the necessary modifications on the elementary level.

\section{REFERENCES}

[1] Aronszajn, N., A unique continuation theorem for solutions of elliptic partial differential equations or inequalities of second order, J. Math. Pures Appl. 36 (1957), 235-249.

[2] Booss-Bavnbek, B., And Wojciechowski, K.P., Elliptic Boundary Problems for Dirac Operators, Birkhäuser, Boston, 1993.

[3] Booss-Bavnbek, B., The unique continuation property for Dirac operators - revisited, Geometry and topology: Aarhus (1998), Contemp. Math. 258 (2000), 21-32.

[4] Booss, B., Eindeutige Fortsetzbarkeit für elliptische Operatoren und ihre formal Adjungierten, Diplomarbeit, Bonn, 1965.

[5] Carey, A.L., And Wang B.L., Notes on Seiberg-Witten-Floer theory. Geometry and topology: Aarhus (1998), Contemp. Math. 258 (2000), 71-85.

[6] Carleman, T., Sur les systèmes linéaires aux dérivées partielles du premier ordre à deux variables, Comptes Rendus 197 (1933), 471-474.

[7] Carleman, T., Sur un problème d'unicité pour les systèmes d'équations aux dérivées partielles à deux variables indépendentes, Ark. Mat. Astr. Fys. 26 B, No 17 (1939), 1-9. 
[8] Cordes, H.O., Über die eindeutige Bestimmtheit der Lösungen elliptischer Differentialgleichungen durch Anfangsvorgaben, Nachr. Akad. Wiss. Göttingen Math.-Phys. Kl. IIa, Nr. 11 (1956), $239-258$.

[9] Floer, A., The unregularized gradient flow of the symplectic action. Comm. Pure Appl. Math. 41 No 6 (1988), 775-813.

[10] Frøyshov, K., The Seiberg-Witten equations and four-manifolds with boundary. Math. Res. Lett. 3 No 3 (1996), 373-390.

[11] Kronheimer, P.B., Embedded surfaces and gauge theory in three and four dimensions, Surveys in differential geometry, Vol. III (Cambridge, MA, 1996), 243-298, Int. Press, Boston, MA, 1998.

[12] Kronheimer, P.B., And Mrowka, T.S., The Genus of Embedded Surfaces in the Projective Plane, Math. Research Lett. 1 (1994), 797-808;

[13] Manolescu, C., Seiberg-Witten-Floer stable homotopy type of three-manifolds with $b_{1}=0$, Preprint arXiv:math.DG/0104024.

[14] Marcoldi, M., AND Wang, B.L., Equivariant Seiberg-Witten Floer homology, Communications in Analysis and Geometry 9 No 3 (2001) 451-639.

[15] Nirenberg, L., Lectures on Linear Partial Differential Equations, Conference Board of the Mathematical Sciences (CBMS), Regional Conference Series in Mathematics no. 17, Amer. Math. Soc., Providence R.I., 1973.

[16] Schwartz, L. Ecuaciones differenciales elipticas, Bogota, Universidad nacional de Colombia, 1956.

[17] Treves, F., Pseudodifferential and Fourier Integral Operators I, Plenum Press, New York, 1980.

[18] WeCK, N., Unique continuation for some systems of partial differential equations, Applicable Analysis 13 (1982), 53-63.

Bernhelm Booss-Bavnbek: Institut for matematik og fysik, Roskilde University, 4000 Roskilde, Denmark

E-mail address: booss@mmf.ruc.dk

Matilde Marcolli: Max-Planck Institut für Mathematik, Vivatsgasse 7, 53111 Bonn, GERMANY

E-mail address: marcolli@mpim-bonn.mpg.de

Bai-Ling Wang: Department of Pure Mathematics, University of Adelaide, AdeLAIDE SA 5005, Australia

E-mail address: bwang@maths.adelaide.edu.au 